\title{
ON THE ASYMPTOTIC DENSITY OF THE $k$-FREE INTEGERS
}

H. M. STARK

A positive integer is said to be $k$-free $(k \geqq 2)$ if it contains no perfect $k$ th power factor greater than 1 . Let $S(x)$ be the number of $k$ free integers $\leqq x$. It is well known that

$$
\delta_{k}=\lim _{x \rightarrow \infty} \frac{S(x)}{x}=\frac{1}{\zeta(k)},
$$

where $\zeta(k)$ is the Riemann zeta function. In fact, Evelyn and Linfoot [2] have shown that

$$
\begin{aligned}
T(x) & =S(x)-\frac{[x]}{\zeta(k)} \\
& =O\left(x^{1 / k} \exp \left[-b(\log x \log \log x)^{1 / 2}\right]\right), \quad b=a k^{-3 / 2}
\end{aligned}
$$

and $a$ is an absolute constant $>0$. They have also shown that

$$
T(x) \neq o\left(x^{1 / 2 k}\right) .
$$

The elementary result that $T(x)=O\left(x^{1 / k}\right)$ goes back to at least 1885 $[3$, p. 47].

Recently, some interest has been shown in the Schnirelmann density of the $k$-free integers:

$$
D_{k}=\inf _{n>0} \frac{S(n)}{n} .
$$

Duncan [5] has shown that

$$
D_{2} \leqq \delta_{2}<D_{3} \leqq \delta_{3}<\cdots<D_{k} \leqq \delta_{k}<D_{k+1} \leqq \cdots .
$$

Since $S(n) / n$ is initially greater than $\delta_{k}$, one may reasonably ask if $D_{k}=\delta_{k}$. Rogers [6] has shown that for $k=2$ this is not so, and in fact

$$
D_{2}=\frac{53}{88}<\frac{6}{\pi^{2}}=\delta_{2}
$$

The method used in [6] is computational and sheds no light on the possible equality of $D_{k}$ and $\delta_{k}$ for $k>2$. We show here that $D_{k}<\delta_{k}$

Received by the editors December 20, 1965. 
as a corollary of the fact that $T(x)$ has infinitely many changes of sign; this in turn is a corollary of

Theorem 1. Let $\rho_{j}=\frac{1}{2}+i \gamma_{j}(j=1,2)$ denote the first two zeros of $\zeta(s)$ above the $\sigma$ axis $\left(\gamma_{1} \approx 14, \gamma_{2} \approx 21\right)$. Let $\alpha_{1}=\zeta\left(\rho_{1} / k\right) /\left[\rho_{1} \zeta^{\prime}\left(\rho_{1}\right)\right]$ and let $L=2\left(1-\gamma_{1} / \gamma_{2}\right)\left|\alpha_{1}\right|>0$. Then

$$
\liminf _{x \rightarrow \infty} x^{-1 / 2 k} T(x) \leqq-L \quad \text { and } \quad \limsup _{x \rightarrow \infty} x^{-1 / 2 k} T(x) \geqq L .
$$

Before we prove Theorem 1 , it is convenient to introduce some notation. Let $b_{n}=a_{n}-1 / \zeta(k)$, where $a_{n}=1$ or 0 according as $n$ is $k$ free or not. Then

$$
T(x)=\sum_{n \leq x} b_{n}
$$

and

$$
\sum_{n=1}^{\infty} b_{n} n^{-s}=\frac{\zeta(s)}{\zeta(k s)}-\frac{\zeta(s)}{\zeta(k)},
$$

where the series converges for $\sigma>1 / k$ by (1).

The proof of Theorem 1 is based on a theorem of Ingham [1]:

THEOREM. Let

$$
F(s)=\int_{0}^{\infty} A(u) e^{-s u} d u,
$$

where $A(u)$ is absolutely integrable over every finite interval $0 \leqq u \leqq U$, and the integral is convergent in some half plane $\sigma>\sigma_{1} \geqq 0$.

Let $A^{*}(u)$ be a real trigonometric polynomial,

$$
\begin{aligned}
A^{*}(u) & =\sum_{n=-N}^{N} \alpha_{n} \exp \left(i t_{n} u\right) \\
& =\alpha_{0}+2 \operatorname{Re} \sum_{n=1}^{N} \alpha_{n} \exp \left(i t_{n} u\right) \quad\left(t_{n} \text { real, } t_{-n}=-t_{n}, \alpha_{-n}=\bar{\alpha}_{n}\right),
\end{aligned}
$$

and let

$$
F^{*}(s)=\int_{0}^{\infty} A^{*}(u) e^{-s u} d u=\sum_{n=-N}^{N} \frac{\alpha_{n}}{s-i t_{n}} \quad(\sigma>0) .
$$

Suppose that $F(s)-F^{*}(s)$ can be continued as an analytic function throughout some domain containing the region $\sigma \geqq 0,-T \leqq t \leqq T$ for some fixed $T>0$. Then, 


$$
\liminf _{u \rightarrow \infty} A(u) \leqq \liminf _{u \rightarrow \infty} A_{T}^{*}(u) \leqq \limsup _{u \rightarrow \infty} A_{T}^{*}(u) \leqq \limsup _{u \rightarrow \infty} A(u),
$$

where

$$
\begin{aligned}
A_{T}^{*}(u) & =\sum_{\left|t_{n}\right|<T}\left[1-\left(\left|t_{n}\right| / T\right)\right] \alpha_{n} \exp \left(i t_{n} u\right) \\
& =\alpha_{0}+2 \operatorname{Re} \sum_{0<t_{n}<T}\left(1-t_{n} / T\right) \alpha_{n} \exp \left(i t_{n} u\right) .
\end{aligned}
$$

To apply Ingham's theorem, let

$$
A(u)=e^{-u / 2 k} T\left(e^{u}\right) .
$$

From (3), (4), and (5), we see that

$$
F(s)=\frac{2 k}{2 k s+1}\left[\frac{\zeta(s+1 / 2 k)}{\zeta(k s+1 / 2)}-\frac{\zeta(s+1 / 2 k)}{\zeta(k)}\right] .
$$

Let

$$
A^{*}(u)=2 \operatorname{Re} \sum_{n=1}^{2} \alpha_{n} \exp \left(i \gamma_{n} u / k\right), \text { where } \alpha_{n}=\operatorname{Re}_{s=i \gamma_{n} / k} s F(s) \quad(n=1,2) .
$$

Finally, let $T=\gamma_{2} / k$ so that

$$
A_{T}^{*}(u)=2 \operatorname{Re}\left[\left(1-\gamma_{1} / \gamma_{2}\right) \alpha_{1} \exp \left(i \gamma_{1} u / k\right)\right] .
$$

Theorem 1 is now an immediate consequence of Ingham's theorem.

In closing, it should be noted that the gap between (1) and Theorem 1 will be hard to close; from (2) and (3), we see that $T(x)$ $=O\left(x^{\sigma / k}\right)$ for all $\sigma>1 / 2$ implies that $\zeta(s)$ has no zeros in the half plane $\sigma>1 / 2$. In the reverse direction, the best known result on the Riemann hypothesis is Axer's result [4],

$$
T(x)=O\left(x^{(2+\epsilon) /(2 k+1)}\right) .
$$

Thus, even with the Riemann hypothesis, the order of $T(x)$ remains in question.

Professor Bateman has noted that a slightly weaker form of Theorem 1 follows from Landau's theorem. Suppose that for some real $c$ and positive $\alpha, T(x)+c x^{\alpha}$ has the same sign for $x>x_{0}$. Then Landau's theorem applied to the formula

$$
s \int_{1}^{\infty} \frac{T(x)+c x^{\alpha}}{x^{s+1}} d x=\frac{\zeta(s)}{\zeta(k s)}-\frac{\zeta(s)}{\zeta(k)}+\frac{c s}{s-\alpha}
$$


says that the function on the right has no singularities in the half plane $\sigma>\alpha$ since the function is regular on the part of the $\sigma$ axis with $\sigma>\alpha$. Since there is a singularity at $\rho_{1} / k$, this is a contradiction if $\alpha<1 /(2 k)$. Thus $T(x)+c x^{\alpha}$ changes sign infinitely of ten for any real $c$ and any $\alpha<1 /(2 k)$.

\section{REFERENCES}

1. A. E. Ingham, On two conjectures in the theory of numbers, Amer. J. Math. 64 (1942), 313-319.

2. C. J. A. Evelyn and E. H. Linfoot, On a problem in the additive theory of numbers. IV, Ann. of Math. (2) 32 (1931), 261-270.

3. F. Gegenbauer, Asymptotische Gesetze der Zahlentheorie, Denk. Akad. Wiss. Wien 49 (1885), 37-80.

4. A. Axer, Über einige Grenzwertsätze, S.-B. Akad. Wiss. Wien (2a) 120 (1911), 1253-1298.

5. R. L. Duncan, The Schnirelmann density of the $k$-free integers, Proc. Amer. Math. Soc. 16 (1965), 1090-1091.

6. Kenneth Rogers, The Schnirelmann density of the squarefree integers, Proc. Amer. Math. Soc. 15 (1964), 515-516.

UNIVERSITY OF MichigAN 